SysCon 2008 - IEEE International Systems Conference

Montreal, Canada, April 7-10, 2008

\title{
Profiling Complex Systems
}

\author{
Renee Stevens \\ The MITRE Corporation \\ 7515 Colshire Drive \\ McLean, Virginia 22102-7539 \\ Phone: +1-703-983-6013, Fax: +1-703-983-5432, Email: stevensr@mitre.org
}

\begin{abstract}
The Department of Defense, like other government agencies and indeed the global business community, faces increasingly complex challenges that cannot be met by stand-alone systems. This has led to growing reliance on increasingly interoperable and interdependent systems that combine multiple organizational and functional capabilities to achieve and overarching mission. This is the motivation for developing systemsof-systems, enterprise systems, and even extended enterprise systems. This paper focuses on the engineering of this class of systems: a process that demands consideration of increasing scale, the rapid pace of change of the underlying technologies, the complexity of system interactions, and, perhaps most important, shared ownership and control. We hypothesize that engineering these systems is inherently different from engineering large-scale but essentially deterministic systems. Decisions about the system(s) under development have to consider not only the technical and programmatic dimensions but also the political, operational and economic contexts. This paper presents a diagnostic tool for profiling complexity and uncertainty in large scale system engineering developments and provides some lessons learned from its application. On the basis of these insights, we propose an approach to tailoring engineering and acquisition strategies and practices to the specific circumstances at hand.
\end{abstract}

Keywords - System-of-systems engineering, enterprise-systems engineering, profiling systems, complex systems, managing uncertainty

\section{INTRODUCTION}

There is growing recognition both in academia and among systems engineering practitioners that the engineering of large-scale complex systems is inherently different from the engineering of well bounded, essentially predictable systems. This has been described as a problem of "nested complexity" in that we are dealing with complex physical systems that are themselves embedded in a complex policy system [1]. This problem becomes even more acute when we deal with systems at the enterprise scale or those that require the cooperative behavior of multiple independent organizations to achieve desired goals and objectives. Decisions about the system(s) under development have to consider not only the technical and programmatic dimensions but also the political, operational and economic contexts.

\section{INCREASING AGILITY, INCREASING INTEROPERABILITY}

Richly networked joint and coalition forces, capable of operating at high tempos and able to adapt to and leverage opportunities as they emerge, are hallmarks of the emerging military force. The commercial world values similar characteristics. The ability to sense, process and make midcourse corrections in response to real-time intelligence is a competitive advantage not just in combat but also in business. In the Department of Defense, we talk about "coherently joint"; in the commercial world, the term is the "extended enterprise." In both cases, we look to information technologies to interconnect a wide range of entities military elements, government agencies or corporations - and provide them with the timely information necessary for adaptive, agile response.

These operational drivers contribute to a growing emphasis on the engineering, development and evolution of rapidly evolving, large-scale, massively interconnected systems intended to bridge traditional organizational, functional, and system boundaries. The practice of systems engineering has evolved over the past half century and will continue to evolve to meet the opportunities challenges of this new class of systems. Traditional processes and practices must be reexamined for their suitability and, where appropriate, new ones should be proposed, vetted and adopted.

A key premise of this paper is that the engineering of these large scales, complex systems must take into account the specific characteristics of the system and the context in which it is being engineered, developed and acquired and in which it will operate. The first step is to be able to characterize the systems in its context. We discuss a Profiler tool which has been developed to help in understanding the system context. The second step is to be tailor practices and approaches to the specifics of the system's circumstances.

\section{PROFILING COMPLEX SYSTEMS}

The Enterprise Systems Engineering Profiler builds on ideas presented by Michael Jackson and P. Keys. [2] Focusing on operational research techniques, the authors argue that system-based problem-solving methodologies 
should be selected based on the context of the problem at issue. To help in choosing the methodology, the authors go on to propose a classification scheme that takes into account two key dimensions of the problem context: the nature of the decision makers and the nature of the system itself. Dvir, Shenhar and Alkaher [3] also introduce a multidimensional framework for distinguishing among projects based on their levels of uncertainty, complexity and pace and argue that projects with different characteristics warrant different management and organizational styles. Subsequent work by Dvir and Shenhar [4] expands the framework to specifically include the dimension of technology. DeMeyer, Loch and Pich [5] recommend the creation of project uncertainty profiles and tailoring the management role and style to the degree of uncertainty.

The notion of defining the problem context along multiple dimensions provides the intellectual basis for the Profiler. The concept of matching problem-solving techniques to the particular problem context underlies our efforts to understand which processes and techniques of traditional systems engineering still apply to the world of large-scale, complex systems and to initiate the process of defining new ones, where these are needed.

The Systems Engineering Profiler is shown in Figure 1. It is designed to be a self-assessment tool that can help the systems engineer and program manager understand the nature and context of the program/project of interest. It is also intended as the basis of a situational model that would help in selecting and adapting the processes, tools, and techniques most applicable to the particular problem and its context. The challenge is to understand the situation sufficiently well to select the most appropriate ones and to adjust the processes and tools as the situation changes over time.

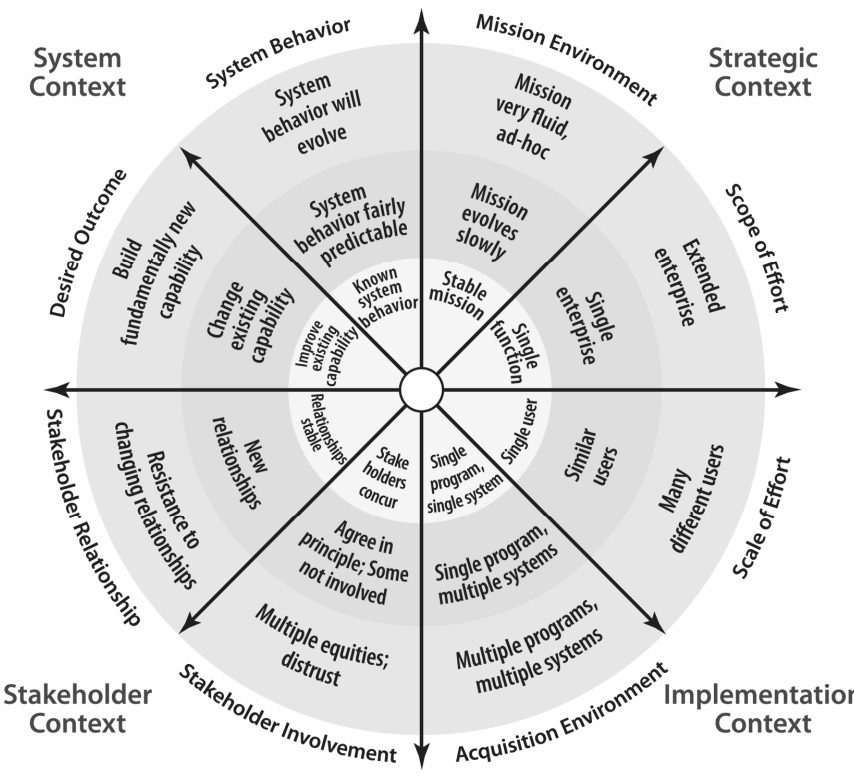

Fig. 1 The Enterprise Systems Engineering Profiler

The Profiler is organized into four quadrants and three rings. The quadrants describe different dimensions of the broader context in which the system, system-of-systems, or enterprise-wide system will be developed, will operate and evolve. The three concentric rings reflect increasing levels of complexity and uncertainty

\section{A. Quadrants}

Reading clockwise, the first quadrant addresses the strategic context. Here the focus is on dimensions related to the stability of the mission environment and the scope and breadth of the intended effort. Requirements for systems that are to operate in a stable environment are expected to change more slowly than those for systems that will operate in environments that are themselves changing. More narrowly focused efforts address a single function. As they broaden, they can be expected to address an enterprise or, in some instances, and extended enterprise.

The second quadrant - the implementation context highlights differences in the scale of the effort - the extent to which the program is expected to support a similar community of interest or to span multiple such communities - and well as its structure. This context can range, at its simplest, from a single program that is established to implement a single system to the obviously more complicated activities associated with multiple programs organized to implement multiple, though operationally interrelated systems.

The third quadrant is the stakeholder context. In this model, we have differentiated two aspects of stakeholder involvement: the extent to which stakeholders agree with the goals and objectives of the effort and the extent to which stakeholder relationships are changing. It is not only the changing relationships that shape the environment but also the extent to which stakeholders accede to or resist such changes.

The fourth quadrant is the systems context. Here the focus is on the expected outcome of the effort as well as on the behavior of the system itself. The expected outcome can range from modest improvements to an existing capability to, at the other extreme, the development of a fundamentally new capability, often by leveraging emerging technologies. The behavior of the system, described primarily in terms of its predictability, is closely related to the expected outcome. Efforts directed toward improving an existing capability are more likely to demonstrate predictable behavior while those focused on developing a fundamentally new capability are also likely to result in behavior that is less predictable and more likely to evolve. The maturity of the technologies to be used in the system is a contributing factor in this quadrant. The performance and interactions of technologies that are at the state of the practice should be well understood while those that are in development or still under exploration will be far less predictable. 


\section{B. Rings}

As one moves out from the origin, the concentric rings reflect increasing complexity, uncertainty, and variability. The innermost ring is the domain of traditional program management and traditional systems engineering, in which the manager and the systems engineer operate inside the program. Here, the effort is most often characterized by wellbounded problems, predictable behavior, and a stable environment.

The middle band can be considered the transitional domain. This is the region of end-to-end systems engineering in which the systems engineer primarily works across system and program boundaries. Here the engineer is likely to exercise influence than direct control.

By contrast, the outermost band - which we have termed the "messy frontier" highlights situations where these managers must deal with a highly fluid environment, distributed development activities often in the absence of a global blueprint, multiple stakeholders with independent, sometimes conflicting equities, and systems whose technical behavior is expected to evolve over time.

\section{Polar Diagram}

Using the Profiler yields a polar diagram (Figure 2). As in the example below, it is possible to have different dimensions in different concentric rings, some in the innermost, others in the middle ring, and still others in the outermost ring. It is important to note that the resulting profile applies to a particular point in time. As the situation changes over time, so would the profile.

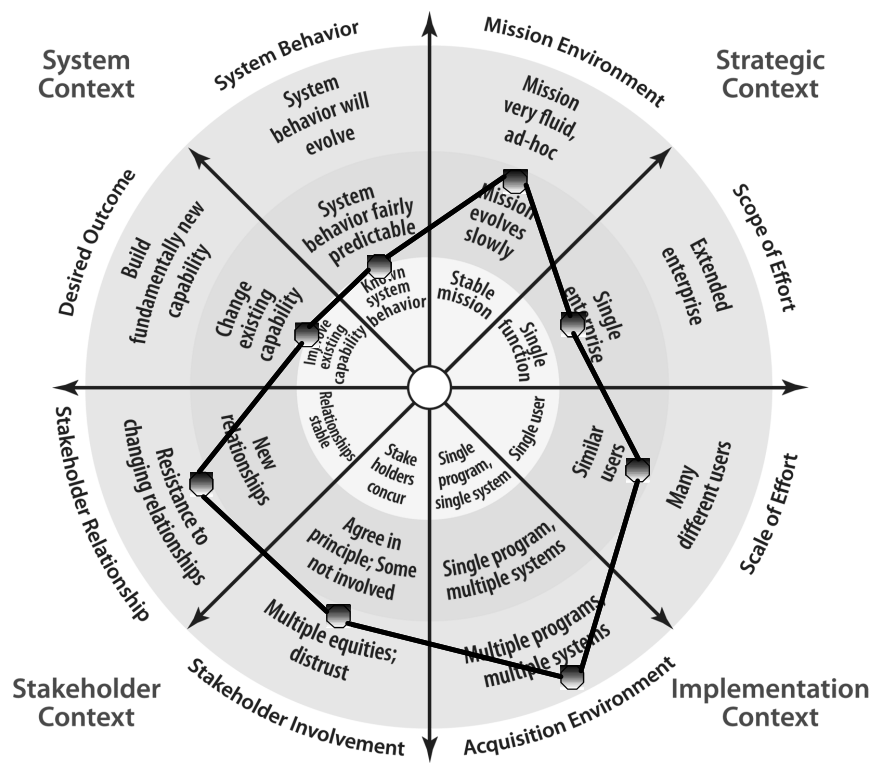

Fig. 2 Using the Profiler to Characterize the Situation

\section{MATCHING SYSTEMS ENGINEERING APPROACHES TO THE SITUATION}

Once there is a profile of the current situation in its multiple dimensions, it becomes possible to match systems engineering and program management strategies and approaches to the specific situation.

\section{A. Strategic context}

For example, if the mission environment in which the system of interest is expected to operate is changing rapidly either in terms of the participants and their interactions or in terms of the underlying business practices, then it is likely that the requirements for the system(s) are also subject to redefinition. In such circumstances, it would be counterproductive to lock in requirements at the beginning and expect them to remain valid and unchanged throughout the duration of the effort. Rather, it would make sense to adopt an iterative and incremental strategy that allows for smaller increments of delivered capability, the opportunity to get critical user feedback and to adapt the next iteration accordingly. In fact, the more volatile the environment, the more frequent the increments. Such a strategy would allow the program to address unanticipated users, not-yet-defined features and to accommodate emerging technologies.

For situations where the system is intended to cross multiple seams, such as in the case of systems that are intended to work across an enterprise or to link strategic partners in an extended enterprise, particularly when these partners lack a history of working effectively with one another, there is merit in structuring the acquisition strategy to first focus on pilot activities. Such pilot activities would address a selected slice of the overall effort and would be directed as much to building trust as to addressing substantive issues of terminology, operational patterns and desired features.

\section{B. Implementation context}

In the implementation context, the more separately managed systems that are required to work collaboratively to provide the needed capability, the more emphasis should be placed on defining the common design patterns, the minimum set of agreed to standards and recommended best practices. For example, situations in which there are multiple legacy systems and where the nature of the interactions between them is difficult to fully anticipate would be best served by design patterns that emphasize flexibility and adaptability. Loose coupling is such an approach to designing resilient interfaces. It limits interdependencies among components and is intended to reduce the risk that changes in one component will yield unanticipated changes in others. In contrast, situations that depend on high levels of synchronization would be best suited to tight coupling design patterns. 


\section{Stakeholder context}

In the stakeholder context, the greater the degree of diversity among the key stakeholders, the more important it is that the engineering and program management understand the positions of each of the key stakeholders and actively work to identify areas of potential intersection. Techniques such as stakeholder analysis become more critical. Bringing stakeholders into the process, such as by engaging them in trade-off analyses, offer opportunities to develop acceptable strategies. In cases where there are divergent stakeholder positions, it may not be possible to meet all of their separate requirements, but it would be critical to identify the intersecting set and establish that as the priority effort.

\section{System context}

In the system context, the more novel the effort, the greater the likelihood that it will be difficult to predict, with any degree of confidence, the behavior of the system until it is developed and deployed. Systems which incorporate technologies which are not yet mature are also vulnerable to expected behaviors. In such circumstances, the systems engineering strategy should emphasize the full range of early and continuous discovery opportunities, including early prototyping, exploratory integration test-beds, field trials and experiments. These venues provide useful insight into the interactions among the elements of the system under development as well as the interactions between the system and its anticipated users.

\section{THE PROFILER AS A POTENTIAL LEADING INDICATOR}

Failure to match practices to the circumstances at hand risks introducing unnecessary friction points in the project's execution. For example, if the mission environment is changing rapidly but the program continues to focus on the documented and approved requirements and fends off changes as "requirements creep", then it risks delivering a capability that may meet contractual requirements but has a high probability of failing to satisfy user expectations. In such cases, it is not uncommon for the system to be rejected outright or nominally accepted but effectively ignored.

Figure 3 shows a notional alignment between the situation as captured by the Profiler and the strategies and practices that are being followed by the program.

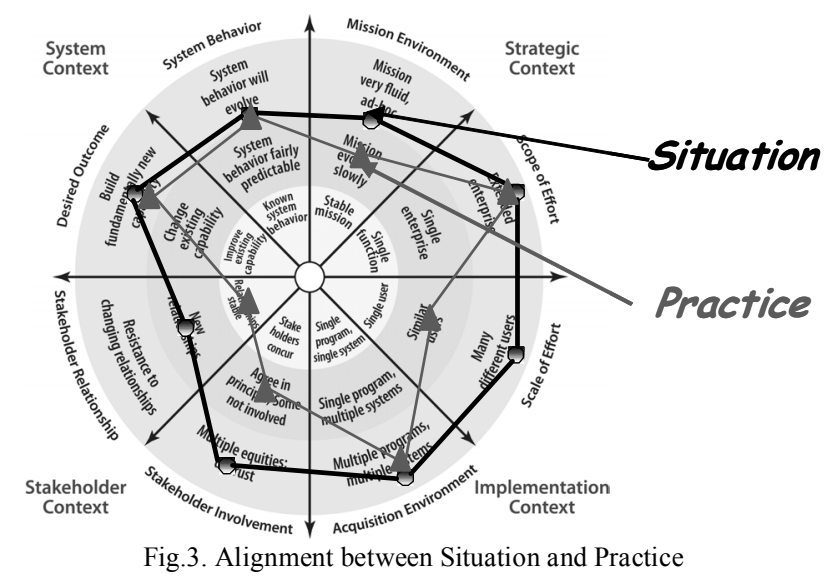

While the degree of divergence between what the situation calls for and the practices that are, in fact, being followed is no guarantee of a successful outcome, one can hypothesize that significant divergence is an indicator of program misalignment in that it introduces unnecessary friction points in the program's execution. As such, the degree of divergence can be viewed as a leading indicator of program health. The greater the total divergence, taking into account both the delta in any single wedge as well as the total number of affected wedges, the greater the magnitude and the breadth of challenges that the program will need to overcome.

Programs where there is little uncertainty and volatility in user requirements and expectations and where the technologies are mature and their performance well understood are best served by detailed planning and then monitoring execution relative to the plan. Programs where requirements are evolving, particularly due to changing conditions in the user's environment, would be best served by staged commitment strategies that allow for adjustments to the target and/or the implementation approach. In contrast, programs in which the general vision is known but where there are alternative potential approaches to realizing that vision may be best served by variation and selection strategies.

While there is a growing body of literature that addresses the need to match the management and engineering style to the project type, considerable research is needed to identify the specific techniques and practices that are best suited to different regimes of the Profiler.

\section{APPLYING THE PROFILER}

The Profiler has been used widely within MITRE to characterize the environment of the programs that we support and to better understand the aspects of complexity that these programs contend with. Through research, project activities and a growing library of case studies, we continue to map specific practices to regions of the Profiler.

We were also invited to apply the Profiler to a government organization and, based on the characterization, to recommend changes to their enterprise systems engineering 
and management processes. While this agency has major acquisition responsibilities, they are not in the area of information technology. For the agency's leadership, this was an opportunity to try a different approach to articulating and communicating the complex nature of their program both internally and to their oversight organizations.

The study team evolved an approach that entailed using the Profiler as an interview vehicle to capture the perspectives of various stakeholders, both inside and outside the agency. Internal stakeholders were senior managers with responsibility for different aspects of the agency's mission. External stakeholders were responsible for overseeing the agency's acquisition activities. A total of 18 interviews were conducted. The key elements of the approach included:

- Tailor the Profiler to the agency's specific context and terminology by adding explanatory text.

- Meet with senior leadership of the agency to clarify their goals and objectives.

- Develop a protocol for conducting the interviews.

- Work with the agency to identify the set of individuals to be interviewed.

- Conduct a kick-off meeting to familiarize key staff with the objectives of the study as well as key elements of the Profiler.

- Schedule and conduct interviews.

- Assess and synthesize the interview responses highlighting similarities and differences.

- Identify engineering and business practice focus areas, focusing on those falling in the "messy frontier"

- Propose new/modified engineering and business practices.

The interview protocol entailed a brief introduction to the study and the Profiler, followed by a series of questions that stepped through each of the eight wedges. For each wedge, the respondent was asked to mark up a blank Profiler and show both where he/she saw the program's current situation and to also depict anticipated changes over the next three to five years. Changes were indicated by arrows showing the direction of the expected change. The rationale for each profile point and vector was captured by at least two note takers.

After trying several different approaches to integrate the interview results, the study team settled on organizing the responses by organizational role of the respondent: (1) site view as provided by site managers with responsibility for operation of a particular geographic site; (2) project view as provided by managers responsible for the design, development and acquisition activities associated with a particular project; (3) agency view as provided by senior managers and those with an agency wide function; and (4) external view. External views were provided by senior staff with a program oversight role as well as by Congressional staffers. See Figure 4 for the composite profiles.

\section{A. Similarities Among Profiles}

Independent of the respondent's role and perspective, there are common perceptions of the key areas of program complexity and uncertainty. The agency is consistently viewed as operating in the outermost ring in two areas. In the Strategic Context, it is consistently described as an extended enterprise in that its success is dependent on the cooperative actions of organizations that are outside of its sphere of control. In the Stakeholder Context, it is viewed as having to deal with multiple stakeholders who have different and often opposing interests, and who distrust each others' motives and actions. While respondents noted that the situation had improved over the initial baseline, they nevertheless continued to view this dimension as one that continues to perturb agency operations. By contrast, areas that were in or near the middle ring were ones that respondents believed may entail risks, but risks that they could managed by technically, programmatically or operational means.

\section{B. Differences Among Profiles}

At the same time, the study team saw some interesting patterns in the profiles associated with the different categories of respondents. Site managers consistently had the most compact profiles with more points in the innermost and middle rings. Project managers and agency-wide managers tended to have successively more expansive profiles. In effect, the broader the role, the more likely the respondent was to view the program as being more complex and more subject to external influences. This pattern is supported by the observation that site managers were more likely to emphasize technical and operational factors that interrupted operations or otherwise impacted their ability to stay on schedule. They viewed these as disruptive but manageable. Project and agency managers also addressed technical and operational issues but tended to add political risk and economic factors as further considerations. In contrast, external respondents tended to view issues primarily through political and economic lenses. In fact, technical and operational issues were often viewed and judged in terms of their political and economic consequences. Such political and economic factors can, and do, constrain the technical and programmatic options that are available to the program. Options that may be technically feasible and cost effective may not prove viable if they encounter community or political opposition. Similarly, local interests may drive the program to implement technical options that have significant cost and schedule consequences.

These findings highlight the critical influence of the external environment on the successful execution of this agency's mission. These external factors add additional measures of uncertainty into the mix, some of which may be foreseeable while others prove unexpected. 


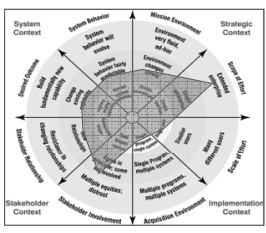

Site Manager View

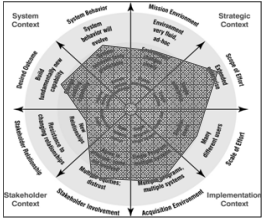

External (Oversight) View

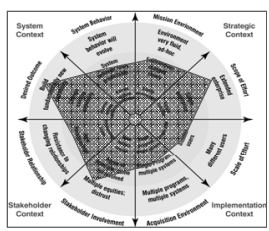

Project Manager View

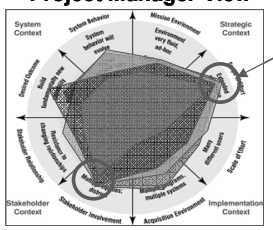

Composite View

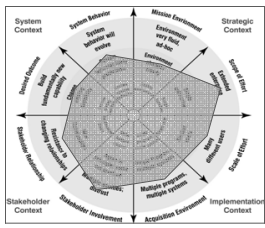

Agency Manager View

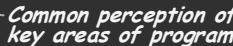

key areas of prograin

uncertainty

Differences relate to

respondent's role and

perspective

Fig. 4 Similarities and Differences among Profiles

\section{SUMMARY}

The Profiler provides a structured approach to characterizing various dimensions of a system, system-ofsystems or enterprise system and highlights factors that contribute to complexity, uncertainty and diversity. Understanding these internal and external influences will help project managers and system engineers to tailor their strategies and practices to the circumstances and to adjust them as circumstances change over time.

\section{REFERENCES}

[1] D.J. Nightingale and D.H. Rhodes, "Enterprise Systems Architecting: Emerging Art and Science within Engineering Systems". MIT Engineering Systems Symposium. Cambridge MA. March 2004.

[2] M. Jackson and P. Keys, "Towards a Sytem-of-systems Methodologies". [sic], J. Opl. Res. Soc., Vol. 35, No. 6, pp 473-486, 1984

[3] D. Dvir, A.J. Shenhar and S. Alkaher, "From a Single Discipline Product to a Multidisciplinary System: Adapting the Right Style to the Right Project". Systems Engineering, Vol. 6, No. 3, pp 123-134, 2003.

[4] A.J. Shenhar and D. Dvir, Reinventing Project Management: The Diamond Approach to Successful Growth and Innovation, Cambridge MA, Harvard Business School Press, 2007.

[5] A. DeMeyer, C.H. Loch and M.T, Pich, "Managing Project Uncertainty: From Variation to Chao". MIT Sloan Management Review. Winter 2002, pp: 60-67.

Copyright $₫ 2008$ by The MITRE Corporation 